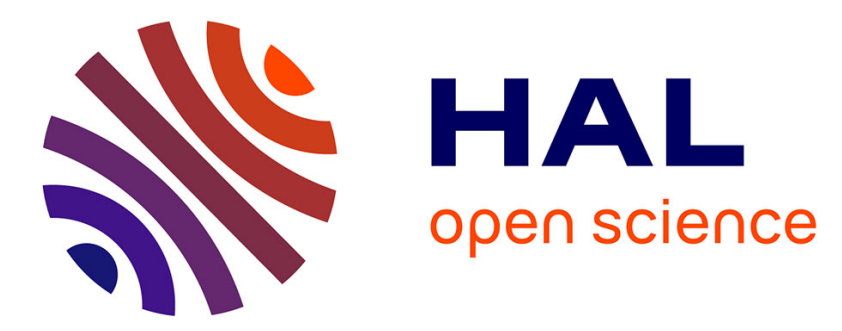

\title{
Construction de la plante de lupin blanc: relations entre la quantité de matière sèche formée et son mode de répartition
}

Alix Pigeaire, Hélène Reponty, Claude Duthion, Vincent Durey, Josette Gonthier, Patrick Mathey

\section{To cite this version:}

Alix Pigeaire, Hélène Reponty, Claude Duthion, Vincent Durey, Josette Gonthier, et al.. Construction de la plante de lupin blanc: relations entre la quantité de matière sèche formée et son mode de répartition. Agronomie, 1985, 5 (10), pp.939-947. hal-00884725

\section{HAL Id: hal-00884725 \\ https://hal.science/hal-00884725}

Submitted on 1 Jan 1985

HAL is a multi-disciplinary open access archive for the deposit and dissemination of scientific research documents, whether they are published or not. The documents may come from teaching and research institutions in France or abroad, or from public or private research centers.
L'archive ouverte pluridisciplinaire HAL, est destinée au dépôt et à la diffusion de documents scientifiques de niveau recherche, publiés ou non, émanant des établissements d'enseignement et de recherche français ou étrangers, des laboratoires publics ou privés. 


\title{
Construction de la plante de lupin blanc : rela- tions entre la quantité de matière sèche formée et son mode de répartition
}

\author{
Alix PIGEAIRE, Hélène REPONTY \& Claude DUTHION \\ avec la collaboration technique de Vincent DUREY, Josette GONTHIER \& Patrick MATHEY
}

I.N.R.A., Laboratoire d'Agronomie, 17, rue Sully, F 21034 Dijon Cedex

Mots clés additionnels : Lupinus albus, structure axiale, nombre de grains. bution of accumulated dry matter.

Vegetative and reproductive organs grow simultaneously during the development of white lupin (Lupinus albus L.). Dry matter partitioning between the aerial parts of plants grown at different densities was studied during the period of competition between emergence of lateral branches and seed setting on the main inflorescence. Partitioning was related to the total amount of dry matter per plant, as follow : lateral emergence failed when earlier dry matter accumulation per plant was below definite thresholds ; the capacity of the different sinks was limited when dry matter accumulation per plant was high.

Additional key words : Lupinus albus, orders of lateral branches, seed number.

\section{INTRODUCTION}

Les variétés de lupin blanc (Lupinus albus L.) actuellement proposées à la culture sont des plantes à floraison de type indéterminé. Les bourgeons situés à l'aisselle des dernières feuilles de l'axe principal donnent naissance à des axes apicaux d'ordre 1 (symbolisés par la suite par A1). Ces axes portent, comme l'axe principal, une inflorescence terminale. Ils peuvent donner eux-mêmes naissance (fig. 1) à des axes d'ordre 2 (A2) et ainsi de suite (FARRINGTON \& GREENWOOD, 1975). Le début d'allongement des axes d'un ordre donné coïncide à quelques jours près avec le début de la floraison sur les axes de l'ordre inférieur (FARRINGTON \& PATE, 1981; AMARGER \& DUTHION, 1984). Les périodes de formation des appareils végétatif et reproducteur sont concomitantes à l'échelle de la plante entière.
La compétition entre les appareils végétatif et reproducteur a été étudiée de différentes manières. Le bilan du carbone à l'intérieur de la plante a été décrit par PATE \& Herridge (1978), à partir de cultures en pots, en conditions d'alimentation optimales. PATE \& FARRINGTON (1981), étudiant les contributions respectives des différentes feuilles-sources à l'alimentation de l'inflorescence principale, ont mis en évidence une très large interdépendance nutritionnelle entre l'axe principal et les axes apicaux. Les résultats d'expériences d'ablation d'organes (MAC GIBBON \& WATKIN Williams, 1980 ; FARrington \& PATE, 1981 ; PORTER, 1982) sont contradictoires selon la densité de peuplement: l'ablation de l'ensemble des axes A1 lorsque commence leur allongement conduit à une augmentation de la production de l'inflorescence principale en densité forte, à sa diminution en densité faible. 


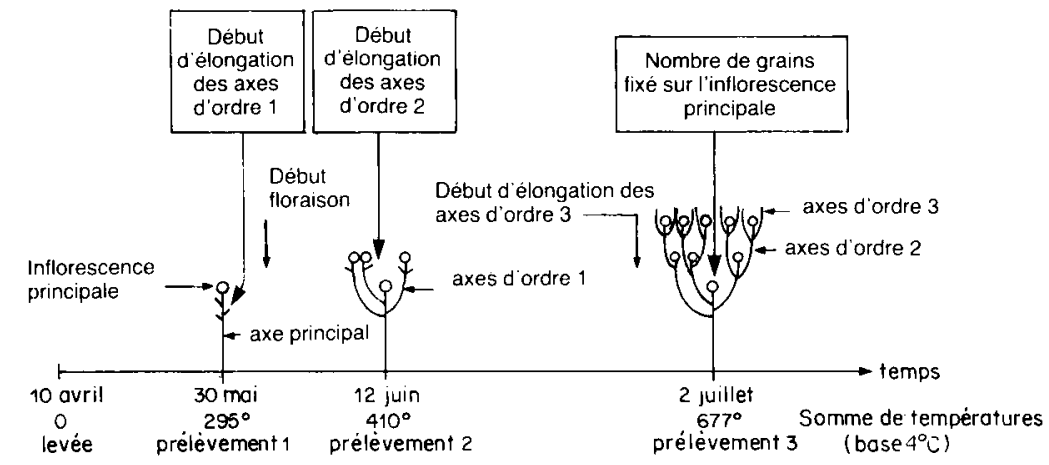

Figure 1

Stade de développement de la plante aux différentes dates de prélèvement.

Le présent travail est une contribution à l'étude des relations de compétition entre les appareils végétatif et reproducteur chez le lupin blanc. Il a pour but de rechercher comment varient les proportions d'assimilats affectées aux différents organes en fonction de la quantité totale d'assimilats produits. Ces proportions ont été décrites dans quelques situations données par PATE \& HERRIDGE (1978) et FARRINGTON \& PATE (1981). Il est très probable qu'elles varient selon les conditions d'alimentation. L'existence d'une hiérarchie entre puits, certains puits étant prioritaires par rapport à d'autres en cas de réduction de la quantité d'assimilats, a déjà été mise en évidence chez une autre légumineuse (modèle de répartition des assimilats chez la luzerne, Gosse et al., 1982). Des connaissances sur cette éventuelle hiérarchie entre puits sont utiles dans la perspective d'une modélisation de l'élaboration du rendement. Il est nécessaire de savoir si le modèle de répartition des assimilats doit être uniquement fonction du stade de développement de la plante ou s'il doit être paramétré, et de quelle façon, par la quantité totale d'assimilats produits.

Cette étude a été abordée à une échelle agronomique en utilisant la matière sèche (MS) comme indicateur des quantités d'assimilats affectées à chaque organe : pour les inflorescences, le nombre de grains non avortés (cf. II-D.2) a constitué un $2^{e}$ indicateur. Les gains de MS par type d'organes ont été analysés en fonction du gain de MS pour la plante entière, pendant des intervalles de temps au cours desquels un ensemble donné d'organes a été en croissance. Une gamme de variation de la MS accumulée par plante a été créée à l'aide d'un traitement « densité de peuplement » dans une expérimentation au champ. La densité de peuplement agit en effet fortement chez le lupin, pour modifier non seulement l'accumulation de MS, mais aussi la structure axiale de la plante, c'est-àdire le nombre, l'identité et l'état de croissance et de développement des axes présents (HERBERT, 1977, 1979). Le travail a été limité aux organes aériens. Il a couvert la phase de compétition entre les appareils végétatif et reproducteur : les observations ont commencé au début de l'allongement des axes A1 et se sont achevées avec la fin de la formation du nombre de grains sur l'inflorescence principale, c'est-à-dire peu de temps après que la plante ait acquis sa structure axiale définitive, avant que ne commence la phase de redistribution des éléments nutritifs au profit des grains.
Stage of development at each sampling date.

\section{MATÉRIELS ET MÉTHODES}

\section{A. Conditions générales de l'essai}

L'expérimentation a été conduite au champ lors de la campagne 1984, à l'aide d'un dispositif expérimental implanté sur un sol brun calcique argileux à Bretenières, près de Dijon. Le sol a été fertilisé avec $120 \mathrm{~kg} / \mathrm{ha}$ de $\mathrm{P}_{2} \mathrm{O}_{5}$ et $\mathrm{K}_{2} \mathrm{O}$. Le désherbage a été réalisé en 2 interventions : traitement en prélevée avec du Tribunil (matière active : méthabenzthiazuron) à la dose de $2,5 \mathrm{~kg} / \mathrm{ha}$ de matière active, puis désherbage manuel un mois après la levée.

Le semis a été effectué le 12 mars, avec la variété «Lublanc ». L'inoculation des graines a été réalisée au cours du semis par apport sur le rang, à raison de $10^{6}$ bactéries environ par graine, d'une suspension d'une souche efficiente de Rhizobium lupini fournie par le Laboratoire I.N.R.A. de Microbiologie des Sols de Dijon. La levée a été totale le 10 avril.

La culture n'a pas été irriguée. L'évapotranspiration maximale (ETM) a été estimée d'après les valeurs de l'évapotranspiration potentielle (ETP) calculées selon la formule de Penman et les coefficients culturaux $(\mathrm{k}=$ ETM/ETP Penman) du lupin blanc déterminés à Dijon sur 5 années d'observation (DUTHION \& DuREY, 1984). Pendant la durée de l'essai, les valeurs de (ETM - P) cumulées depuis la levée (P représentant les précipitations) n'ont dépassé $70 \mathrm{~mm}$ que lors de la dernière décade $(103 \mathrm{~mm})$. La réserve utile du sol est estimée à environ $120 \mathrm{~mm}$ : on peut donc considérer que les plantes observées n'ont pas subi de déficit hydrique.

Les températures moyennes mensuelles ont été inférieures aux températures moyennes des 20 années 1961 à 1981 en ce même lieu (respectivement 8,1 et $8,9^{\circ} \mathrm{C}$ en avril ; 10,1 et $12,8{ }^{\circ} \mathrm{C}$ en mai ; 15,8 et $16,2^{\circ} \mathrm{C}$ en juin). Des températures élevées sont susceptibles de provoquer, chez les différentes espèces de lupins, des avortements d'organes reproducteurs. DOWNES \& GLADSTONES (1984) en particulier ont montré chez le lupin bleu (Lupinus angustifolius L.) que des plantes maintenues quelques jours à une température diurne de $33{ }^{\circ} \mathrm{C}$ ont des nombres de fleurs puis des nombres de grains par gousse significativement réduits. Les plantes observées lors de l'essai n'ont vraisemblablement pas subi d'avortements par excès de température : les températures maximales sont restées faibles, 
ne dépassant $26^{\circ} \mathrm{C}$ qu'en 3 occasions, sans jamais atteindre $30^{\circ} \mathrm{C}$.

\section{B. Traitements appliqués}

- La gamme de variation de l'accumulation de MS par individu a été créée à l'aide de différentes densités de peuplement (tabl. 1). Cinq de ces densités (de 35 à 115 plantes $/ \mathrm{m}^{2}$ ) ont été réalisées au semis, la $6^{\mathrm{e}}$ a été obtenue en éclaircissant manuellement un peuplement à 35 plantes $/ \mathrm{m}^{2}$, dès que la levée a été terminée. Les densités réelles obtenues, mesurées sur 6 fois $1 \mathrm{~m}$ linéaire sur chaque parcelle, sont reportées dans le tableau 1. Le dispositif expérimental comporte 2 blocs. Chaque parcelle comporte 6 lignes de $16 \mathrm{~m}$ de long, espacées de $30 \mathrm{~cm}$.

- Le traitement " densité de peuplement "n'est ici qu'un moyen commode de créer une gamme de variation de la MS accumulée par plante et non l'objet du travail présenté. Les mêmes densités peuvent conduire à des accumulations différentes selon les lieux et les années. Leur seule énumération est par conséquent insuffisante pour caractériser de façon correcte le traitement expérimental «MS accumulée par plante». Elle est complétée par les données du tableau 1: valeurs moyennes, écarts types, minimum et maximum de la MS par plante, aux 3 dates de prélèvement (la définition de ces dates figure dans le paragraphe suivant). A la date (1), les traitements ne sont pas différenciés. Ils le sont aux dates (2) et (3).

Dans les conditions de l'essai, l'effet des traitements «densité de peuplement » peut être interprété comme provenant principalement des modalités de la compétition pour la lumière. Il n'y a pas eu en effet de déficit hydrique (§ II-A). Il a été vérifié par ailleurs que l'inoculation avait été efficace. AMARGER \& DUTHION
(1984) ont montré que, dans ce cas, la fixation symbiotique de l'azote permet un ajustement de la quantité totale d'azote accumulée aux besoins de la plante exprimés en fonction de sa matière sèche. Enfin, aucun symptôme de carence minérale n'a été enregistré.

A maturité, les rendements obtenus ne sont pas significativement différents (seuil 5 p. 100) ; ils sont relativement faibles, variant de 28 (densité $15 \mathrm{pl} / \mathrm{m}^{2}$ ) à 32 (densité $95 \mathrm{pl} / \mathrm{m}^{2}$ ) $\mathrm{q} / \mathrm{ha}$ de grains secs. Des accidents climatiques (grêle, tornades) sont survenus après le dernier prélèvement, occasionnant des dégâts d'autant plus importants que la densité était plus élevée.

\section{Prélèvements}

\section{Dates}

La figure 1 représente le stade de développement de la plante pour les 3 dates de prélèvement retenues. Pour les critères choisis, il n'y a pas de différences de stade de développement entre densités.

Les 2 premières dates marquent l'apparition d'un nouveau type d'organes pour la plante : respectivement les axes $\mathrm{A} 1$ et $\mathrm{A} 2$. La convention qui a été adoptée pour déterminer le début d'allongement des axes est une longueur moyenne (mesure effectuée quotidiennement sur 20 plantes de chacune des 2 densités extrêmes) de $1 \mathrm{~cm}$ pour les axes les plus longs de l'ordre considéré. Tous les axes d'un même ordre commencent leur allongement à des dates voisines.

La dernière date est proche de celle à laquelle le nombre de grains de l'inflorescence principale est fixé. Il existe chez le lupin, comme chez d'autres légumineuses, un stade limite pour l'avortement des grains

TABLEAU

Densités de peuplement, nombres de plantes prélevées et matières sèches par plante pour les différents traitements. Les dates de prélèvement sont indiquées en figure 1.

Plant density, number of sampled plants and dry matter per plant for the different treatments. Sampling dates as in figure 1 .

\begin{tabular}{|c|c|c|c|c|c|c|c|}
\hline \multicolumn{2}{|c|}{ Numéro du traitement } & 1 & 2 & 3 & 4 & 5 & 6 \\
\hline \multicolumn{2}{|c|}{ Densité souhaitée (plantes $/ \mathrm{m}^{2}$ ) } & 15 & 35 & 55 & 75 & 95 & 115 \\
\hline \multicolumn{2}{|c|}{ Densité obtenue (plantes $/ \mathrm{m}^{2}$ ) } & 16 & 36 & 50 & 64 & 86 & 99 \\
\hline \multirow{3}{*}{$\begin{array}{l}\text { Nombre de } \\
\text { plantes } \\
\text { prélevées } \\
\text { (total : } 711 \text { ) }\end{array}$} & Prélèvement 1 & 10 & 26 & 29 & 47 & 51 & 64 \\
\hline & Prélèvement 2 & 7 & 25 & 30 & 42 & 61 & 74 \\
\hline & Prélèvement 3 & 8 & 22 & 37 & 50 & 55 & 73 \\
\hline \multirow{3}{*}{$\begin{array}{l}\text { Matière sèche } \\
\text { par plante }(\mathrm{g}) \\
\text { Moy. } \pm \text { écart type } \\
\text { (1) }\end{array}$} & $\begin{array}{l}\text { Prélèvement } 1 \\
\text { Min. } 0,28 \text { Max. } \quad 3,25\end{array}$ & $\begin{array}{c}1,79 \pm 0,73 \\
\mathrm{ab}\end{array}$ & $\begin{array}{c}2,16 \pm 0,45 \\
\mathrm{a}\end{array}$ & $2,25(2)$ & $\frac{2,00 \pm 0}{a}$ & 1,64 & $1,78 \pm \frac{ \pm}{b} 0,54$ \\
\hline & $\begin{array}{c}\text { Prélèvement } 2 \\
\text { Min. } 0,65 \operatorname{Max} .12,01\end{array}$ & $\underset{\mathrm{a}}{7,42 \pm 2,37}$ & $\begin{array}{c}6,47 \pm 2,06 \\
a\end{array}$ & 5,90 & $4,97 \underset{b}{\mathrm{~b}}$ & 4,14 & $\begin{array}{c}4,21 \pm 1,29 \\
\mathrm{c}\end{array}$ \\
\hline & $\begin{array}{c}\text { Prélèvement } 3 \\
\text { Min. } 0,88 \text { Max. } 38,77\end{array}$ & $\begin{array}{c}28,34 \pm 8,17 \\
a\end{array}$ & $\begin{array}{c}14,19 \pm 3,98 \\
b\end{array}$ & 9,37 & $\begin{array}{c}8,94 \pm 3,47 \\
\mathrm{c}\end{array}$ & 7,38 & $6,96 \pm 3,51$ \\
\hline
\end{tabular}

(1) A une même date, les traitements qui ne sont pas suivis de la même lettre sont significativement différents au seuil 5 \%0 (Test de Student). (2) Ecart type non déterminé ; sur les densités 3 et 5 , les pesées n'ont pas été faites individuellement pour chaque plante mais globalement pour l'ensemble du traitement. 
qui peut être repéré chez cette espèce par une longueur du grain de $9 \mathrm{~mm}$ (PIGEAIRE et al., 1986). Le $3^{\mathrm{e}}$ prélèvement a été effectué quand la moyenne (mesure effectuée quotidiennement sur 20 plantes de chacune des 2 densités extrêmes) des longueurs des grains les plus longs dans la dernière gousse de l'inflorescence principale est égale à $5 \mathrm{~mm}$. Il est postérieur au début d'allongement des axes A3 quand celui-ci a eu lieu.

\section{Echantillonnage}

Les plantes ont été prélevées sur une placette par parcelle (soit 2 placettes par traitement). Chaque placette mesure $50 \mathrm{~cm}$ de long et comprend les 2 lignes centrales de la parcelle. Les effectifs des plantes prélevées selon les dates et les densités sont reportés dans le tableau 1.
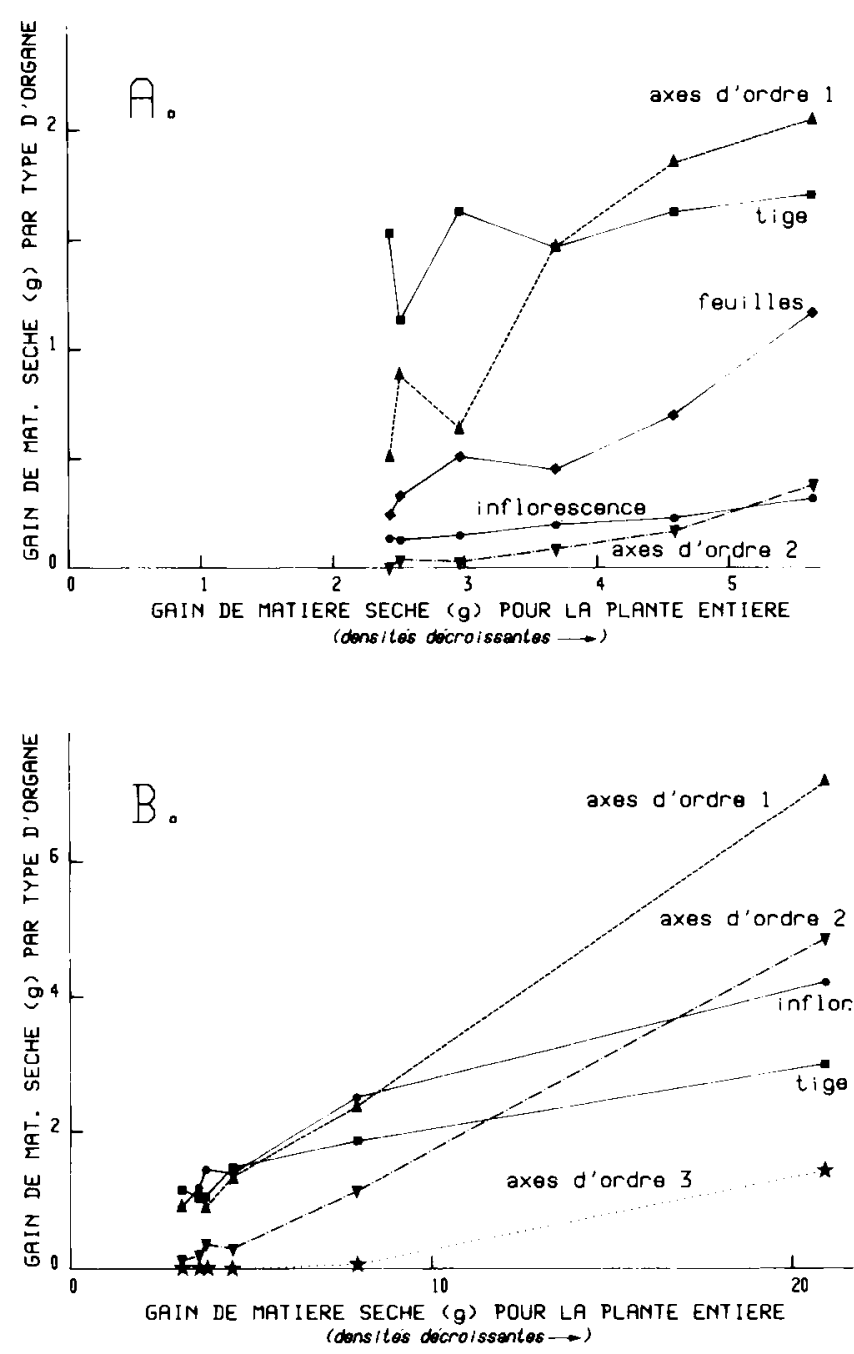

Figure 2

Gains moyens de MS par type d'organes en fonction du gain moyen de MS pour la plante entière; (A) entre les dates (1) et (2),

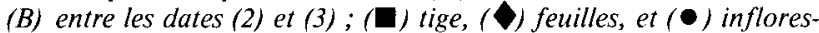
cence de l'axe principal; $(\mathbf{\Delta})$ axes d'ordre $1,(\boldsymbol{\nabla})$ axes d'ordre 2 , (*) axes d'ordre 3.

Observed mean dry matter gain per plant and per plant part; (A) between dates (I) and (2), (B) between dates (2) and (3); (D) stem, (-) leaves, and (O) inflorescence of the main axis;

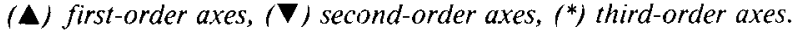

\section{Mesures}

\section{Poids secs par type d'organes}

Le découpage par type d'organes a été fait d'une façon qui privilégie l'étude de la compétition entre les axes apicaux et l'inflorescence principale, sans chercher à faire la distinction entre feuilles, tige et inflorescence dans le cas des axes apicaux. Ce découpage est le suivant : tige de l'axe principal - feuilles de l'axe principal - inflorescence principale - axes A1, un par un selon leur rang - axes A2, regroupés selon le rang de l'axe A1 qui les porte - axes A3, regroupés selon le rang de l'axe A2 qui les porte.

Pour les densités $1,2,4$ et 6 , les pesées sont faites de façon individualisée pour chaque plante ; pour les densités 3 et 5 , elles sont faites globalement pour l'ensemble des plantes de la même densité.

\section{Nombre de grains de l'inflorescence principale}

Le nombre de grains d'une longueur supérieure à $5 \mathrm{~mm}$ - c'est-à-dire à 15 p. 100 près par excès, le nombre de grains non avortés (PIGEAIRE et al., 1986) - a été mesuré à la date 3 sur les inflorescences principales. Dans la plupart des cas, ce nombre a pu être observé par transparence à travers la gousse. Pour les cas litigieux, les gousses ont été ouvertes et les grains mesurés.

Le nombre de grains non avortés est un indicateur de la quantité d'assimilats reçue par l'inflorescence principale. Avant le franchissement du stade limite d'avortement, ces derniers ont 2 utilisations principales : la croissance des enveloppes de gousses, accessoirement celle du racème de l'inflorescence, et la réalisation des divisions cellulaires dans les jeunes graines; on considère généralement que l'arrêt précoce de ces divisions, c'est-à-dire l'avortement des graines, a pour principale origine un défaut de fourniture en assimilats. Ces 2 utilisations sont fortement liées l'une à l'autre : les gousses ne s'allongent que si au moins un de leurs grains se développe et leur allongement est plus ou moins proportionnel au nombre des grains qui se développent.

\section{RÉSULTATS}

\section{A. Relations entre la quantité de matière sèche for- mée et son mode de distribution dans la plante}

Dans l'exposé des résultats, la matière sèche de l'inflorescence sera utilisée comme unique indicateur de la quantité d'assimilats reçue par les inflorescences principales : à la $3^{\mathrm{e}}$ date de prélèvement, elle est en effet fortement corrélée avec le nombre $(x)$ des grains d'une longueur supérieure à $5 \mathrm{~mm}$ :

$$
\operatorname{MS}(\mathrm{g})=0,11 \mathrm{x}+0,13 ; \mathrm{r}=0,937 .
$$

Les gains moyens de MS par type d'organes, exprimés en fonction du gain moyen pour la plante entière, sont représentés en figure $2 \mathrm{~A}$ et $\mathrm{B}$, respectivement pour les intervalles de temps compris entre les dates (1) et (2) $\left(13 \mathrm{j} ; 115^{\circ}\right.$.j) et entre les dates (2) et (3) 
$\left(20 \mathrm{j} ; 267^{\circ} . \mathrm{j}\right)$. Chacune des valeurs des abscisses correspond à une densité de peuplement. Les différentes densités sont classées par ordre décroissant : les gains de MS par plante sont d'autant plus élevés que la densité de peuplement est plus faible.

Les courbes représentatives des différents types d'organes ne forment pas un faisceau de droites passant par l'origine : la répartition de la MS ne s'effectue pas toujours dans les mêmes proportions mais varie selon la quantité totale formée. Les différentes courbes se distinguent selon 2 aspects principaux :

- Pour la plupart des organes, l'ordonnée correspondant à la densité la plus forte n'est pas nulle. Les axes $\mathrm{A} 2$ en figure $2 \mathrm{~A}$ et les axes A3 en figure $2 \mathrm{~B}$ font exception : lorsque les gains de MS par plante sont faibles, certains des axes ne commencent pas leur croissance. Ces axes constituent par conséquent des puits non prioritaires.

- Quelques-unes des courbes s'apparentent à des droites, croissantes avec le gain de MS par plante. Les autres par contre s'infléchissent aux faibles densités : ainsi, pour les axes d'ordre 1 sur la figure $2 \mathrm{~A}$, l'inflorescence principale et la tige de l'axe principal sur la figure $2 \mathrm{~B}$. La tendance est encore plus nette pour la tige de l'axe principal en figure $2 \mathrm{~A}$ dont la courbe représentative est presque parallèle à l'axe des abscisses alors que son extrapolation vers les faibles gains de MS doit passer nécessairement par l'origine. La portion de courbe tracée manifeste donc l'existence d'un plateau, atteint dès les plus fortes densités. L'existence de valeurs-plateaux peut être interprétée comme une limitation de la capacité de certains organes à stocker de la matière sèche.

Entre les dates (2) et (3), le gain de MS de la tige de l'axe principal n'est plus limité. L'augmentation des capacités de stockage de la tige correspond à la formation de tissus secondaires (O’NEILL, 1961). Le poids par unité de longueur de tige commence à augmenter quand la tige a terminé son allongement, soit une dizaine de jours après le début de la floraison (FARRINGTON \& PATE, 1981). Nous avons pu vérifier qu'à la date (2), l'axe principal avait atteint sa longueur définitive sur toutes les densités.

L'absence d'émission de certains axes et la limitation de la capacité de certains puits sont responsables des variations observées dans la répartition de la matière sèche. L'hétérogénéité entre plantes d'un même traitement (tabl. 1) conduit à poursuivre l'analyse de ces 2 phénomènes, et notamment des valeurs seuils qu'ils mettent en jeu, non pas à partir des données par plante moyenne, mais en utilisant les données obtenues pour chaque plante prise individuellement, sur les densités $1,2,4$ et 6 . Les données moyennes ne permettent pas en effet de mettre en évidence des valeurs seuils significatives par rapport au fonctionnement de la plante. Les causes de l'hétérogénéité entre plantes d'un même traitement ne sont pas connues. Elles peuvent être liées à la variabilité génétique au sein d'une même variété, à des différences initiales pour le poids des graines semées ou pour la date de levée, ou à une variabilité ultérieure des conditions de micro-environnement propre à chaque plante. Il est très probable que les résultats présentés ci-dessous ne sont pas indépendants de la nature des causes ayant effectivement créé la variabilité de la matière sèche par plante observée au sein de chaque traitement.

\section{B. Relations entre la matière sèche de la plante et sa structure axiale}

\section{Matière sèche de la plante et émission des axes}

Les figures $3 \mathrm{~A}$, B et $\mathrm{C}$ représentent les nombres d'axes A1, A2 et A3 en fonction de la MS de la plante, respectivement aux dates de prélèvements (1), (2) et (3). Les nombres d'axes d'un ordre donné sont ainsi confrontés à la MS qu'avait la plante à une date proche du moment de leur formation : à la $1^{\text {re }}$ date de prélèvement, le nombre final d'axes A1 était atteint pour toutes les densités, de même pour les axes A3 à la date (3) ; par contre, pour les axes A2 à la date (2), il ne l'était que sur les densités 3 à 6 . Dans tous les cas, la contribution des axes nouvellement formés au poids total de la plante était encore très faible : en moyenne $7,7-1,4$ et 0,3 p. 100 respectivement aux dates (1), (2) et (3). A chaque date, la variabilité de la MS totale par plante n'a pas pour principale origine les différences de MS des axes nouvellement formés. La gamme de variation de ces derniers (maximum moins minimum de MS) ne représente, par rapport à la gamme de variation pour la plante entière, que 13 , 7 et 8 p. 100 respectivement aux dates (1), (2) et (3).

Les 3 nuages de points obtenus (fig. 3) présentent des caractéristiques communes :

- Il existe une valeur seuil de MS par plante, en dessous de laquelle aucun axe n'est émis. Cette valeur est de $0,8 \mathrm{~g}$ pour les axes $\mathrm{Al}$ à la date (1), 3,0 g pour les axes A2 à la date (2) et $10,2 \mathrm{~g}$ pour les axes A3 à la date (3). Un seul axe A4 a été obtenu. Le poids sec de la plante le portant est de 21,6 g (date 3 ). Une seule exception à cette règle s'observe en figure $3 \mathrm{~B}$ (point A). Il s'agit d'une plante n'ayant que 2 axes $\mathrm{A} 1$, qui a émis 4 axes $\mathrm{A} 2$ alors que sa MS est inférieure au seuil de $3,0 \mathrm{~g}$.

- Au-dessus de cette valeur seuil, le nombre d'axes A1 varie de 1 à 6 , le nombre d'axes $\mathrm{A} 2$ de 0 à 14 et le nombre d'axes A3 de 0 à 19 . Les nombres d'axes les plus élevés sont atteints pour des valeurs de MS par plante de plus en plus grandes. Il existe une très grande variabilité $d u$ nombre d'axes par plante à l'intérieur de chacun des traitements (dispersion des points des figures $3 \mathrm{~A}, \mathrm{~B}, \mathrm{C}$ ) et cette variabilité n'est pas liée à l'hétérogénéité de l'accumulation de MS par plante. Chez d'autres espèces, le rôle de l'éclairement (OIZUMI, 1962, pour le soja) et l'implication du phytochrome (LETOUZÉ, 1972, pour le saule) dans le démarrage de croissance des bourgeons axillaires ont été mis en évidence. Rien toutefois ici ne permet de suspecter une hétérogénéité de l'éclairement des plantes d'un même peuplement pouvant entraîner un écart de 1 à 6 pour les axes d'ordre 1 . Une autre hypothèse explicative pourrait être la variabilité entre plantes du nombre de bourgeons initiés sur les différents axes; elle n'a pu être vérifiée, faute des observations nécessaires.

Pour les axes A2 et A3, des nuages de points d'une allure identique à ceux des figures 3 sont obtenus si l'on met en abscisses, non plus le poids total de la plante, mais seulement le poids des axes porteurs. Les valeurs seuils de MS pour l'émission des axes A2 et A3 sont alors respectivement de $250 \mathrm{mg}$ pour le total des axes Al à la date (2) et de $350 \mathrm{mg}$ pour le total des axes A2 à la date (3). 

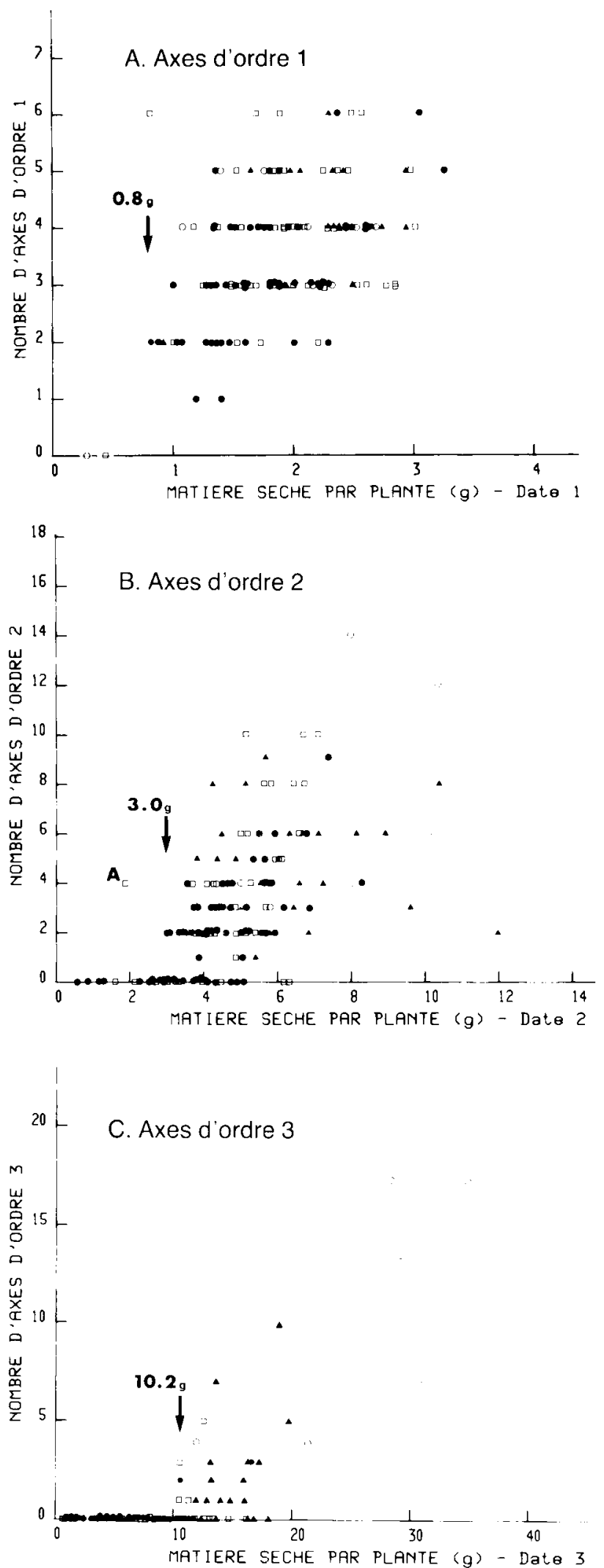

Figure 3

Nombres d'axes des ordres 1,2 et 3, en fonction de la MS de la plante à la date de début d'allongement des axes de l'ordre considéré ( $A$ et $B$ ) ou à une date légèrement postérieure $(C)$; densités I

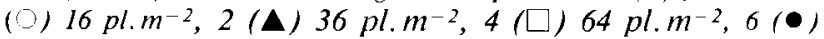
$99 \mathrm{pl} . \mathrm{m}^{-2}$. Chaque point représente une plante. Les flèches indiquent les valeurs seuils de MS par plante pour l'émission des axes.

Number of first, second and third-order axes and plant dry weight at the beginning of emergence of axes of the considered order ( $A$ and $B$ )

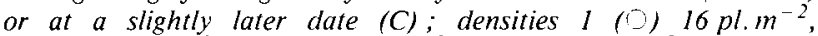
2 (४) $36 \mathrm{pl} . \mathrm{m}^{-2}, 4$ ( $\square$ ) $64 \mathrm{pl} . \mathrm{m}^{-2}, 6(\bullet) 99 \mathrm{pl} . \mathrm{m}^{-2}$. Each spot represents one plant. Arrows indicate the dry matter thresholds for lateral order emergence.
Nous avons pu observer que l'absence d'émission des axes d'un ordre donné se produit quand les axes de l'ordre inférieur n'achèvent pas leur croissance et ne fleurissent pas, mais aussi parfois lorsque ceux-ci sont complets et ont commencé à fleurir. Pour les axes A2, 38 p. 100 des plantes qui n'en portent pas, sont des plantes sur lesquelles au moins un axe Al a fleuri.

\section{Matière sèche par ordre d'axes}

La quantité totale de MS d'un ordre d'axes est indépendante, le plus souvent, du nombre des axes qu'il contient. Ce point est illustré par les figures $4 \mathrm{~A}$ et $\mathrm{B}$ qui représentent, à la date (3), la MS totale des axes respectivement $\mathrm{A} 1$ et $\mathrm{A} 2$ en fonction de leur nombre. Sur ces figures, les nuages de points correspondant aux densités 2,4 et 6 ont une forme allongée dans le sens de l'axe des abscisses : il n'existe pas,
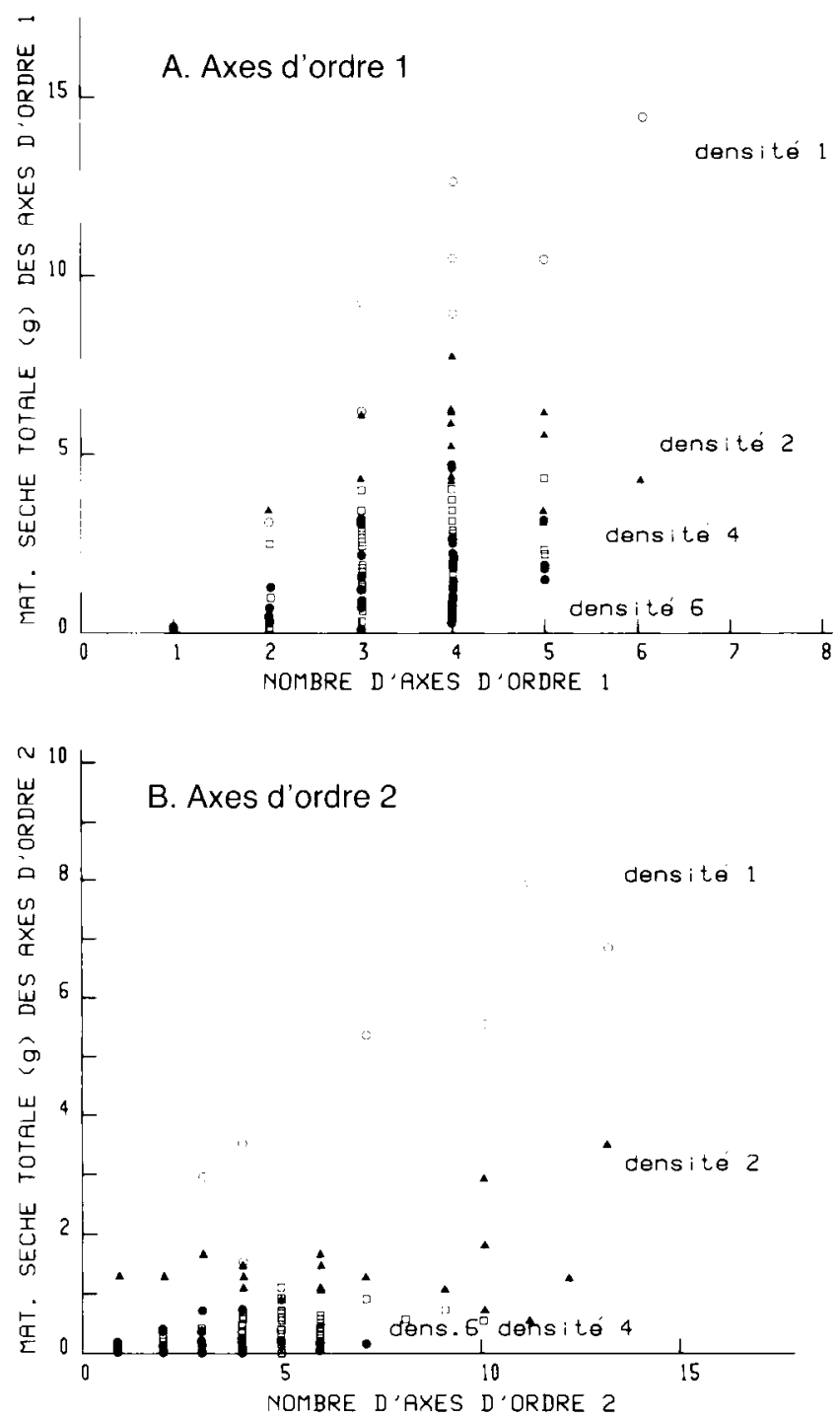

Figure 4

Nombres d'axes d'un ordre donné et MS totale de ces axes, plante par plante, à la date (3). (A) axes d'ordre $1,(B)$ axes d'ordre 2;

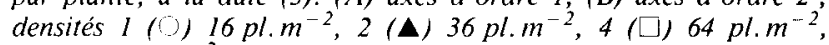
$6(\bullet) 99 \mathrm{pl} . \mathrm{m}^{-2}$. Chaque point représente une plante.

Number of axes and total dry matter per order; $3 r d$ sampling date. (A) first-order axes (B) second-order axes ; densities 1 ( $O$ ) $16 \mathrm{pl} . \mathrm{m}^{-2}, 2$ (ム) $36 \mathrm{pl} . \mathrm{m}^{-2}, 4$ (口) $64 \mathrm{pl} . \mathrm{m}^{-2}, 6(\bullet) 99 \mathrm{pl} . \mathrm{m}^{-2}$. Each spot represents one plant. 
pour ces densités, de relation entre le nombre d'axes et leur poids total. C'est aussi le cas pour l'ensemble des densités à la date (2). Par contre, dans le cas de la densité 1 à la date (3), on note une relation entre les 2 variables. Cette relation forme, pour les points des autres densités, une courbe enveloppe dont l'existence indique que l'accumulation de MS par axe est limitée : quand la limite est atteinte, le nombre d'axes est le facteur déterminant de la MS totale des axes. Il devient aussi d'ailleurs, celui de la MS totale de la plante. A la date (3), pour la densité 1, le coefficient de corrélation entre la MS de la plante et son nombre d'axes d'ordre 1 est de 0,86 .

Les valeurs limites moyennes de la quantité de MS par axe à la date (3) peuvent être déterminées en calculant pour les points de la densité 1 la pente de la droite de régression passant par l'origine. Ces valeurs limites moyennes sont égales à $2,43 \mathrm{~g}$ pour les axes A1 et $0,58 \mathrm{~g}$ pour les axes $\mathrm{A} 2$. Il existe en fait un gradient selon le rang de l'axe : pour les axes A1 par exemple, ceux qui sont situés directement sous l'inflorescence principale ont les poids les plus bas $(1,69 \mathrm{~g})$; les axes situés aux nœuds 2,3 et 4 ont les poids les plus élevés $(2,81 ; 2,67 ; 2,83 \mathrm{~g})$; les axes de rang 5 et 6 ont des poids intermédiaires $(2,09 ; 2,25 \mathrm{~g})$. Ce gradient diffère de celui observé par HERBERT (1979) sur une autre variété de lupin blanc (axes de rang 1 et 2 d'un poids supérieur aux axes de rang 3 ).

\section{Limitation de la capacité des puits de l'axe principal}

L'infléchissement des courbes de gains de MS (fig. 2) suggérait une limitation de la capacité des puits de l'axe principal. La figure 5 représente la MS des différents organes de l'axe principal et le nombre de grains de son inflorescence en fonction de la MS

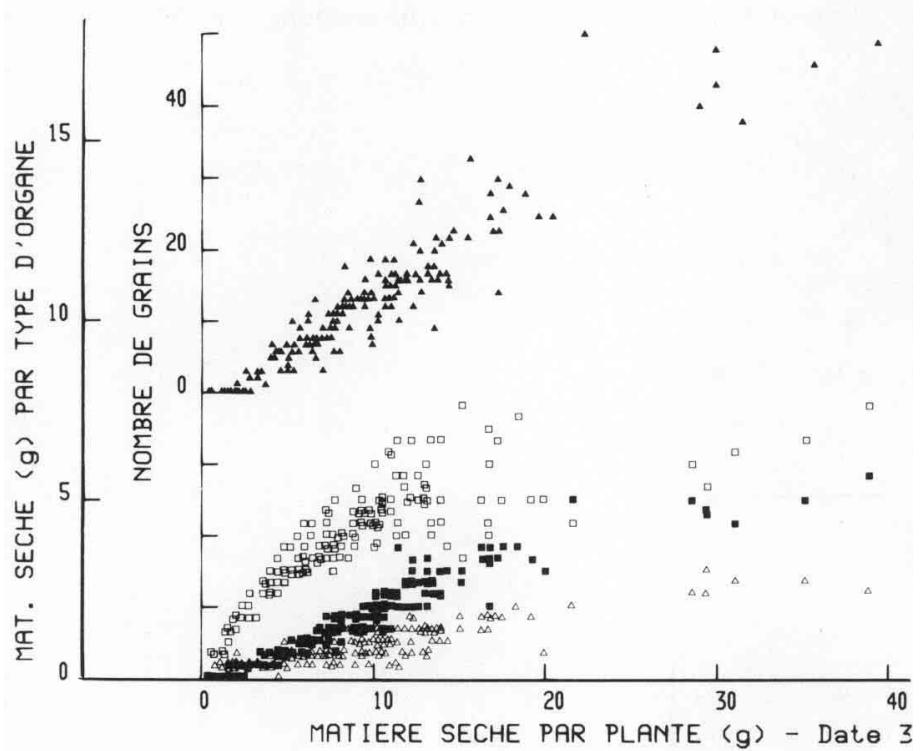

Figure 5

Matières sèches des différents organes de l'axe principal, ( $\square)$ tige, $(\triangle)$ feuilles, $(\mathbf{\square})$ inflorescence et nombre de grains de son inflorescence $(\mathbf{\Delta})$, en fonction de la MS totale de la plante, à la date (3). Chaque point représente une plante.

Dry matter of main stem parts ( $\square$ ) stem, ( $\triangle$ ) leaves, ( $\boldsymbol{\square})$ inflorescence, seed number of main inflorescence $(\mathbf{\Delta})$ and dry matter per plant; 3rd sampling date. Each spot represents one plant. totale de la plante à la date (3). Le faible nombre de points représentatifs de la densité 1 et leur écart avec ceux de la densité 2 sur l'échelle des abscisses compliquent l'analyse. Il semble néanmoins que ces séries de points présentent toutes une tendance à l'infléchissement pour les fortes valeurs de MS par plante. Cette tendance apparaît plus nettement pour la tige et les feuilles de l'axe principal que pour son inflorescence. Elle pourrait indiquer l'existence de valeurs limites de MS qui ne seraient pas encore tout à fait atteintes même sur la plus faible densité. On peut noter, en utilisant la loi de SHINOZAKI \& KIRA (1956), qu'à la date (3), la MS obtenue sur les plantes de la densité 1 ne représente pas la MS maximale potentielle par plante. Cette loi indique en effet qu'à une date donnée, $1 / \mathrm{P}=\mathrm{aD}+\mathrm{b}$, avec $\mathrm{P}$ représentant le poids de MS de la plante et $D$ la densité de peuplement. Appliquée à nos données à la date (3), $\mathrm{a}=0,0128$ et $\mathrm{b}=0,0255$ avec un coefficient de corrélation $\mathrm{R}^{2}=0,94$. Quand $\mathrm{D}$ tend vers $0, \mathrm{P}$ tend vers $1 / \mathrm{b}$ soit $39 \mathrm{~g}$. La MS moyenne par plante sur la densité 1 n'est que de $28 \mathrm{~g}$ (tabl. 1).

\section{DISCUSSION ET CONCLUSIONS}

Cette étude a permis de montrer comment les proportions de MS affectées aux différents puits de la plante varient selon la quantité totale formée par celle-ci. Nous nous proposons de récapituler les résultats obtenus en montrant ce qu'ils peuvent apporter, notamment par rapport à une démarche de diagnostic cultural.

\section{A. Non-émission des axes et diagnostic cultural}

Le gain de MS par ordre d'axes est nul tant que l'accumulation de MS de la plante entière n'a pas dépassé une certaine valeur. Ce résultat, obtenu dans des conditions où la compétition interplante s'exerce principalement vis-à-vis de la lumière ( $\S$ II-B.2) est vraisemblablement généralisable à des situations pour lesquelles le principal facteur limitant est, soit l'eau, soit l'azote. D'après COLLOT (1979), l'intervention d'une contrainte hydrique perturbe l'émission des axes, quand ils sont au début de leur allongement. D'après DUTHION (résultats non publiés), les plantes non inoculées dont la nutrition azotée est limitante ont une structure axiale réduite à l'ordre 1 ou 2 .

Pour certaines céréales, des méthodes de diagnostic cultural ont été élaborées en utilisant les variations des composantes du rendement (blé : SÉBILLOTTE, 1980 ; maïs : NAVARro Garza, 1984). Ces méthodes nécessitent la connaissance des lois de formation des composantes du rendement, afin que le niveau de celles-ci, enregistrées à la récolte, puisse renseigner a posteriori sur le fonctionnement de la plante durant le cycle. Les différentes décompositions du rendement n'ont pas toutes la même pertinence vis-à-vis du diagnostic cultural. Il convient de choisir une décomposition potentiellement riche en informations et, si possible, pas trop coûteuse à obtenir.

Dans le cas du lupin, il apparaît judicieux de retenir une décomposition du rendement par ordre d'axes ; la décomposition par rang d'axe au sein de chaque ordre 
est par contre superflue :

- Tout se passe comme si chaque ordre d'axes constituait une même entité fonctionnelle. Au sein d'un ordre, les différents axes commencent à s'allonger au même moment. Une quantité totale de MS leur est allouée, qui se répartit entre les différents axes en fonction de leur nombre. Le nombre d'axes par ordre varie beaucoup entre plantes d'un même traitement, mais peu entre traitements. Il ne peut pas être utilisé pour discriminer des traitements. Ce sont le nombre d'ordres d'axes et la MS totale (puis la production totale) par ordre d'axes, qui permettent de différencier des traitements.

- La décomposition du rendement par ordre d'axes est nécessaire et suffisante pour classer les organes reproducteurs par date de floraison. Ce classement peut être utile pour détecter des causes d'avortement qui interviendraient de façon momentanée dans le cycle.

La mise en place de la composante « nombre d'ordre d'axes " dépend à la fois de la somme de températures et de l'accumulation de MS par plante. La somme de températures fixe un rythme maximal pour l'émission des ordres d'axes. Ce rythme n'est tenu que si l'accumulation de MS par plante est supérieure à certains seuils. Par conséquent, lorsque le nombre d'ordres d'axes présents sur une plante est inférieur au nombre maximal d'ordres possible pour la variété considérée, il fournit une indication de la MS accumulée par plante. Cette indication reste grossière : la gamme de variation du nombre d'ordres d'axes est faible (1 à 4) ; il peut y avoir absence d'émission des axes d'un ordre donné pour des plantes ayant dépassé les seuils de MS (fig. 3 B et $\mathrm{C}$ ). Pour affiner la mesure de cet indicateur de croissance, il pourrait être utile de définir une échelle de notation indiquant la progression de la plante vers l'émission de l'ordre d'axes suivant. Une telle échelle devrait permettre de transformer la mesure discontinue du nombre d'ordres d'axes émis, en une mesure continue.

\section{B. Nombre maximal de grains de l'inflorescence principale}

Pour les fortes accumulations de MS par plante, il apparaît une limitation à la quantité reçue par les différents organes. Dans le cas de l'inflorescence principale, la MS accumulée pendant la phase précédant le franchissement du stade limite d'avortement est fortement corrélée au nombre de grains formés ( $\S$ III-A). Une limitation de cette MS a donc pour corollaire l'existence d'un nombre de grains limite par inflorescence. Il n'a pas été possible de déterminer si ce nombre limite était atteint sur notre essai (fig. 5). MAC GIBBON \& WATKIN WILliams (1980), dans une expérimentation faisant varier la densité de peuplement, obtiennent des nombres de grains par inflorescence principale très proches pour les 2 densités les plus faibles $\left(21,8\right.$ pour $6 \mathrm{pl} . \mathrm{m}^{-2}$ et 20,3 pour $\left.25 \mathrm{pl} . \mathrm{m}^{-2}\right)$. Ces valeurs sont inférieures aux nombres de grains maximaux obtenus sur notre essai (45-50 grains). Elles illustrent la variabilité du nombre maximal de grains par inflorescence, que l'on pouvait supposer a priori.

\section{Récapitulation des valeurs de matière sèche par plante, qui représentent des valeurs seuils vis-à-vis de son mode de répartition}

Cette récapitulation, effectuée en figure 6 sur un axe représentant la MS de la plante à la date (3), permet de situer les unes par rapport aux autres les différentes valeurs seuils mises en évidence au cours de ce travail et de placer par rapport à elles les MS des plantes correspondant aux densités les plus couramment pratiquées. Les valeurs seuils pour l'émission des axes de différents ordres sont reportées dans la portion supérieure de la figure. Pour les axes A1 et A2, ces valeurs, déterminées à la date (3), sont très peu différentes de celles obtenues aux dates (1) et (2) respectivement (fig. 3). Les plantes qui avaient une MS trop

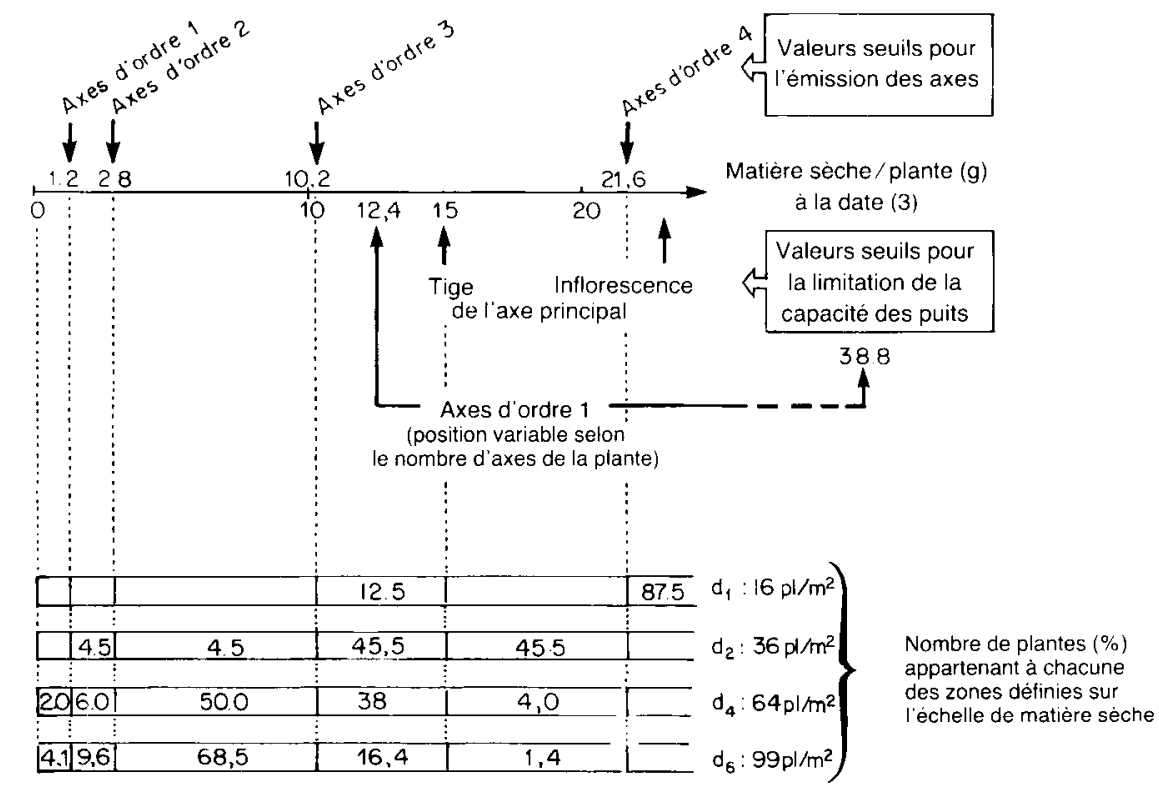

Figure 6

Schéma récapitulatif des valeurs seuils de la MS par plante à la date (3), qui définissent l'absence de certains organes ou la limitation de leur capacité à stocker de la matière sèche. Répartition des plantes des différentes densités sur cette échelle de MS.
Recapitulation of the dry matter thresholds for emergence of lateral orders and for limitation of sink capacity. Distribution of the plants of each density on this scale. 
faible pour permettre l'émission des axes A1 ou A2, n'ont presque pas ou pas du tout poursuivi leur croissance. Les valeurs, à partir desquelles les MS par type d'organes commencent à s'infléchir, sont inscrites sous l'axe de MS par plante. Dans le cas des axes A1 et A2, ces valeurs dépendent du nombre d'axes de la plante. C'est donc une fourchette qui est représentée ; elle correspond à la ganıme de MS des plantes ayant atteint les valeurs limites de MS par axe, c'est-àdire les plantes de la densité 1 ; la borne inférieure $(12,4 \mathrm{~g})$ correspond à la plante qui porte le moins d'axes Al (2), la borne supérieure $(38,8 \mathrm{~g})$, à celle qui en a le plus (6).

Les valeurs reportées sur la figure 6 ne sont pas indépendantes de la date du prélèvement. Par conséquent, elles sont plus intéressantes en tant que valeurs relatives qu'en tant que valeurs absolues. Leurs positions respectives montrent que lorsque la MS par plante augmente, on passe presque sans transition de la zone où certains axes ne sont pas émis à la zone où la capacité de certains organes à stocker la MS commence à être limitée.

La position des plantes des différentes densités est représentée au bas de la figure 6 par le pourcentage des plantes appartenant aux différentes zones définies sur l'échelle de MS. Les densités 2 et 4 sont celles qui encadrent la densité actuellement recommandée par l'ITCF pour la culture du lupin blanc $\left(50 \mathrm{pl} . \mathrm{m}^{-2}\right)$. Pour ces 2 densités, et dans les conditions de l'essai :

- l'émission des axes A2 et surtout A3 est fréquemment limitée. Ceci renforce l'intérêt de la méthode de diagnostic cultural suggérée précédemment ;

- la matière sèche et le nombre de grains de l'inflorescence principale ne sont jamais limités. Dans un modèle de répartition de la MS s'appliquant à des situations culturales courantes, le problème de la limitation du nombre de grains sur l'inflorescence principale ne serait donc vraisemblablement pas à prendre en compte. Toutefois, un nombre maximal de grains plus faible (environ 20 grains) est obtenu dans certaines situations (MAC GIBBON \& WATKIN WILliAMS, 1980). Plus ce nombre est faible, plus il risque d'être atteint à de fortes densités.

Reçu le 22 février 1985. Accepté le 2 août 1985.

\section{RÉFÉRENCES BIBLIOGRAPHIQUES}

Amarger N., Duthion C., 1984. Optimisation des relations solplantes-Rhizobium. Cas de l'association lupin-Rhizobium lupini. Rapport scientifique du contrat de collaboration scientifique entre la CEE et l'I.N.R.A., n ${ }^{\circ}$ CEE 404, 27 p.

Collot H., 1979. Les besoins en eau du lupin blanc. Mémoire de fin d'études ENSSAA-I.N.R.A. Dijon, 38 p.

Downes R. W., Gladstones J. S., 1984. Physiology of growth and seed production in Lupinus angustifolius L. I. Effect on pod and seed set of controlled short duration high temperatures at flowering. Aust. J. Agric. Res., 35, 493-499.

Duthion C., Durey G., 1984. Quelques données à propos de l'alimentation hydrique du lupin blanc, p. 141-147. In «Pourquoi Pois ». Recueil des communications de la journée nationale sur les protéagineux, Paris 12 déc. 1984, ITCF, 311 p.

Farrington P., Greenwood E. A. N., 1975. Description and specification of the branching structure of lupins. Aust. J. Agric. Res., 26, 507-510.

Farrington P., Pate J. S., 1981. Fruit set in Lupinus angustifolius cv Unicrop. I. Phenology and growth during flowering and early fruiting. Aust. J. Plant Physiol., 8, 293-305.

Gosse G., Chartier M., Lemaire G., Guy P., 1982. Influence des facteurs climatiques sur la production de la luzerne. Fourrages, 90, 113-133.

Herbert S. J., 1977. Growth and grain yield of Lupinus albuis at different plant populations. N. Z. J. Agric. Res., 20, 459-465.

Herbert S. J., 1979. Density studies on lupins. I. Flower development. Ann. Bot., 43, 55-63.

Letouzé R., 1972. Phytochrome et croissance du bourgeon axillaire chez Salix babylonica. C. R. Acad. Sci. Paris, 275, 2663-2666.

Mac Gibbon R., Watkin Williams, 1980. Effects of plant and canopy density on seed yield and oil content in white lupin. Expl. Agric., 16, 409-414.

Navarro Garza H., 1984. L'analyse des composantes du rendement du mais. Application à l'étude de la variabilité du rendement dans une petite région. Thèse Doct. Ing., Institut National Agronomique Paris-Grignon, $238 \mathrm{p}$.

Oizumi H., 1962. Studies on the mechanism of branching and its agronomic considerations in soybean plants. Tohoku Nat. Agric. Exp. Stn. Bull., 25, 1-95.

O'Neill T. B., 1961. Primary vascular organization of Lupinus shoot. Bot. Gaz., 123, 1-8.

Pate J. S., Farrington P., 1981. Fruit set in Lupinus angustifolius cv Unicrop. II. Assimilate flow during flowering and early fruiting. Aust. J. Plant physiol., 8, 307-318.

Pate J. S., Herridge D. F., 1978. Partitioning and utilization of net photosynthate in a nodulated annual legume. J. Exp. Bot., 29, 401412.

Pigeaire A., Duthion C., Turc O., 1986. Caractérisation du stade limite d'avortement des grains chez le soja de type indéterminé, le lupin blanc et le pois (accepté pour publication dans Agronomie).

Porter N. G., 1982. Interaction between lateral branch growth and pod set in primary inflorescences of lupin. Aust. J. Agric. Res., 33, 957-965.

Sébillotte M., 1980. An analysis of yield elaboration in wheat, p. 25-32. In E. Hafliger : «Wheat, technical monograph ». CibaGeigy, Bâle, Suisse, 95 p.

Shinozaki K., Kira T., 1956. Intraspecific competition among higher plants. VII. Logistic theory of the C-D effect. J. Inst. Polytech. Osaka City Univ. D7, 35-72. 